\title{
Study of diffusion processes in carrot particles
}

\section{Iryna Huzova, Volodymyr Atamanyuk}

Department of Chemical Engineering, Lviv Polytechnic National University

Bandera street, 12, Lviv, Ukraine, 79013, E-mail: iryna.o.huzova@1pnu.ua

Internal porosity of the carrot particle, the value of which determines the coefficient of mass transfer have been determined. On the basis of the experiments we derived the dependence, allowing to determine the mass transfer coefficients for sucrose molecules in blanched carrot fruits within the temperatures corresponding to the quality of the finished product.

Keywords - diffusion, temperature, internal surface, carrot fruit, sucrose, mass transfer coefficient

\section{Introduction}

Candied fruits is a capillary-porous matter with a complex structure, saturated with sugar. That is why the value of the coefficients of sucrose diffusion in the plant material and proceeding of the diffusion process on the whole will be influenced by the temperature of the sugar syrup, as well as the shape, size and internal structure of the raw material.

\section{Materials and methods}

The carrots were cut into cylinders $12 \mathrm{~mm}$ in diameter and $4 \mathrm{~mm}$ in height. After the fruit was blanched for 10 minutes. The slice was taken from the middle of the cylinder (the part between the core and the bark). A slice of carrot with a thickness of 500 microns was analyzed under a electronic microscope. Eyeglass - 10, increase - 400 .

\section{Results of experimental studies}

The general view of raw and blanched carrot cells is shown in Figs. 1a and 1b, respectively. Raw carrot cells are almost rectangular, pores are clearly defined. After blanching the pumpkin cells are almost round and the pores are much wider, which will contribute to a better diffusion of sucrose molecules to the middle of the carrot particle.

From the picture of blanched carrot (Fig. 1b), the average diameter of the pores between the carrot cells $(\mathrm{d}=3 \mu \mathrm{m})$ and the average cross-sectional area of carrot cell $(620 \mu \mathrm{m} 2)$ were determined.

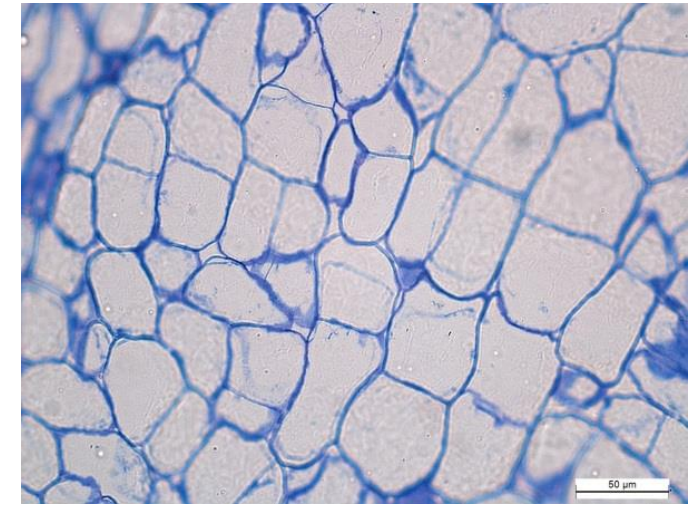

a)

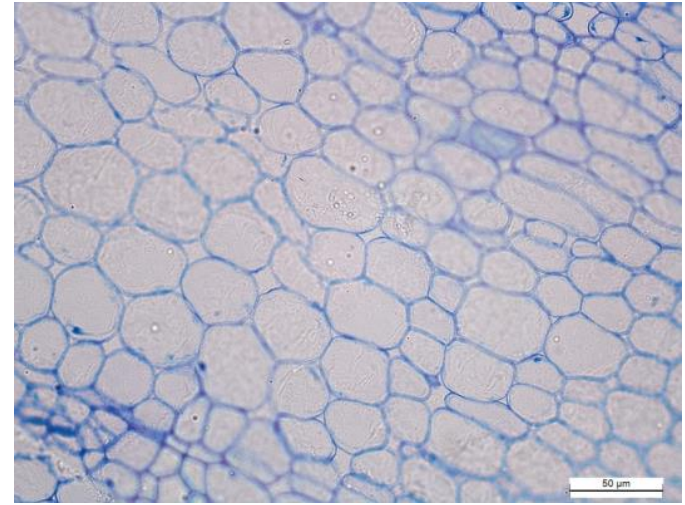

b)

Fig. 1. Photos of the raw (a) and blanched (b) carrot cells

The diffusion coefficient depends on the temperature, nature and shape of the particle. Well-known Einstein's eq. (1) determines the diffusion coefficient of large circular molecules in a liquid [1]: 


$$
D_{c}=\frac{k \cdot T}{6 \cdot \pi \cdot \mu \cdot r}
$$

where $\mathrm{k}$ is Boltzmann constant, $\mathrm{J} / \mathrm{K} ; \mathrm{T}$ is an absolute temperature, $\mathrm{K} ; \mathrm{r}$ is the radius of diffusing particles, $\mathrm{m} ; \mu$ is the viscosity of the liquid medium, $\mathrm{Pa} \cdot \mathrm{sec}$

The radius of diffusing particles is the radius of the sucrose molecule. Taking into account that $D_{c}$ is the coefficient of isothermal diffusion in a liquid continuum, in porous bodies, a coefficient of mass transfer $D_{m}$ is used instead of $D_{c}$.

As a rule, the coefficient $D_{m}$ is less than the diffusion coefficient $D_{c}$. The mass transfer area is less compared with that for free diffusion. The ratio of these areas is equal to the matter porosity $\mathrm{m}$.

Thus, the mass transfer coefficient will be equal to

$$
D_{m}=m \cdot D_{c}
$$

where $m$ is the porosity $\mathrm{m}^{2} / \mathrm{m}^{2}$ $\mathrm{m}^{2} / \mathrm{m}^{2}$.

The porosity is determined from the photograph of the sample slice (Fig. 1b), $m=0.3$

Fig. 2 shows the dependence of the mass transfer coefficient determined from equation (2) on the experimental temperature of the medium. The obtained points were approximated by exponential dependence. As a result of the approximation, the equation (3) is obtained:

$$
D_{m_{T}}=6.62 \cdot 10^{-4} \cdot D_{m_{293}} \cdot e^{0.02 \cdot T}
$$

The values of the coefficients in Eq. (3) depend only on the fruit kind and its internal structure.

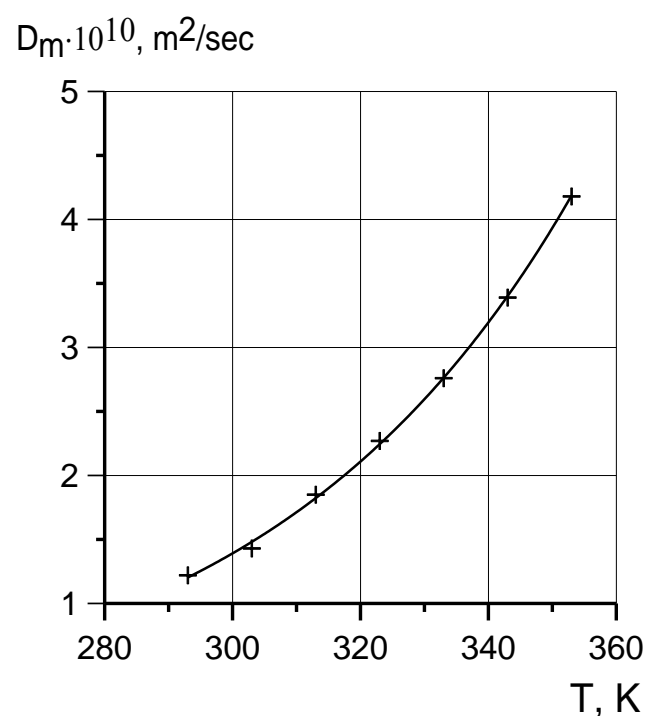

Fig. 2. Dependence of the mass transfer coefficient of sucrose molecules in blanched carrot fruits on the medium temperature.

\section{Conclusion}

Eq. (3) allows to determine the mass transfer coefficients of sucrose molecules in blanched carrot fruits at the temperature corresponding to the quality of the finished product.

\section{References}

[1] Atamanyuk V., Huzova I., Gnativ Z. (2017). Study of diffusion processes in pumpkin particles during candied fruits production. Food Science and Technology, 11(4), 21-28. doi.org/10.15673/fst.v11i4.727 